\title{
RADIO MAPPING
}

\author{
E.R. Deul and W.B. Burton \\ Sterrewacht Leiden \\ P.O. Box 9513, 2300 AA Leiden \\ The Netherlands
}

\begin{abstract}
The mapping discussed in this paper is based on observations obtained from the 21-cm emission line of neutral atomic hydrogen. We will give an overview of the existing large surveys and present a homogeneous datacube that contains the most important surveys. This datacube was created by regridding the original observations, for the surveys included, onto a common denominator grid. Presenting a three dimensional dataset of intensities in one single image is impossible, therefore we made a film that displays in a time sequence a set of cuts (position-velocity maps) through the datacube. The advantage of showing the third coordinate in time is that the eye can now catch continuities in this coordinate that were previously hidden in the data.
\end{abstract}

\section{INTRODUCTION}

The hyperfine transition of neutral atomic hydrogen produces a spectral line at $21 \mathrm{~cm}$ wavelength. Spectra of this line, usually expressed in brightness temperature, show the Doppler shift of this line in terms of radial velocity with respect to the local standard of rest. Mapping a region of the sky we obtain spectra at consecutive points, thus building up a three-dimensional image, with the two mapping coordinates and one velocity coordinate, of that region.

During the past decades several surveys of the galactic neutral atomic hy- 
drogen have been made. Due to the relatively better instruments on the northern hemisphere, over the past decade this part of the sky has been surveyed more frequently by several different instruments at increasingly better resolution and sensitivity than the southern hemisphere. The geographic positions of the instruments are directly linked to the coverage extent of the surveys. To obtain an image of the whole sky one needs to merge a minimum of two different surveys. In practice it is better to use a larger number of surveys because then there will be a considerable overlap on which calibration tests can be performed. We will give a list of the currently existing large scale surveys and discuss the ones implemented in our database.

A major problem with the modern high sensitivity surveys is the stray radiation (Lockman et al., 1986; Kalberla et al., 1980). This effect caused by the relatively weak but still significant sensitivity of a radio telescope at large angles away from the main pointing direction can lead to observed fluxes at low intensity regions that are at maximum $0.5 \mathrm{~K}$ too bright. Although this is not important for the present high galactic latitude surveys it contributes significantly to the more sensitive large-scale galactic-plane surveys.

\section{THE SURVEYS}

Observations of the neutral atomic hydrogen started soon after the hyperfine transition line was predicted by H.C. van de Hulst in 1944. The first observations made in 1951 already gave a good idea of the sky distribution of $\mathrm{HI}$, but the transformation from observed intensity-velocity profiles to the spatial distribution of $\mathrm{HI}$ number density is still a matter of controversy. An important issue in determining the observational constraints for an all-sky survey are the amount of time needed per grid point and the number of grid points in the sky. Typical modern surveys last in the order of one year observing time. This has considerably limited the number of large scale surveys, and has put very strict constraints on the resolution and sensitivity.

Our aim was to create a datacube of currently available HI observations covering the entire sky at a velocity range as wide as possible. We choose a grid in galactic coordinates as the principle coordinates and velocity as the third coordinate such that $(i)$ the spatial gridding is the minimum of the surveys included, and (ii) the velocity coverage is enough to seperate kinematically independent structures. The grid size is $(l, b, \mathrm{v})=\left(0^{\circ} .25,0.25,2.0 \mathrm{~km} \mathrm{~s}^{-1}\right)$.

The surveys included in this dataset are (in alphabetical order): Burton (1986), Cleary et al. (1979), Heiles and Habing (1974), Kerr et al. (1987), and 
Weaver and Williams (1973). Each survey was treated separately and regridded to the final grid points of the large datacube. The interpolation in velocity space was done by deconvolving the profiles with a gaussian of the width used in the survey, then interpolated with a gaussian distribution of $2 \mathrm{~km} \mathrm{~s}^{-1}$ width and finally using a hanning taper to obtain the $2 \mathrm{~km} \mathrm{~s}^{-1}$ velocity grid. In position each survey was sampled on its own grid with unobserved points linearly interpolated. Particularly in the Cleary $e t$ al. and the Heiles and Habing data this resulted in adding up to $20 \%$ interpolated points. Then the final grid resolution was established using a fourpoint Lagrange interpolation function. In the final datacube the regions of overlap contain carefully weighted means of the surveys covering that region. Weights are based on the telescope resolution, the survey sensitivity and the sampling positions of the original data.

The final datacube consists of surface brightnesses as a function of three coordinates $I(l, b, v)$ and contains $(1440,480,170)$ datapoints equivalent to $470 \mathrm{Mb}$. On request the data is available on magnetic tape in FITS format.

To enable display of this 3-D dataset we have made a film, showing planar cuts through the cube at a constant third coordinate. Consecutive frames of the film show planar cuts at a gradually increasing third coordinate, such that when displayed the film presents the complete dataset in a time sequence. For the film we have choosen $(b, v)$ cuts at constant longitude, because they are easy to interpret. Figure 1 shows the sample frame of the film. As a compromise between the display of the complete galactic latitudes range, missing the small scale structure at low latitude and a small subset of latitudes around the galactic plane, missing the larger scale structure at higher latitudes we have choosen to clip the latitude range between $-20^{\circ}$ and $+22^{\circ}$. The complete velocity extend of the datacube is shown. In the top right-hand corner of the panel a disc with radius $15 \mathrm{kpc}$ is shown depicting the top view of our galaxy. The cross at the center of the disc marks the galactic center and a line is drawn from the position of the sun along the line-of-sight in the direction of the longitude at which the frame displays the planar cut. This line gradually rotates as the film proceeds in time. The intensities are displayed on a logarithmic scale to enhance the low level structure; a constant grading is used throughout. The film starts at $l=180^{\circ}$ and moves through the third and fourth quadrant with increasing length of the line-of-sight. Then it gradually moves through the galactic center region to the first and second quadrant to finish at the starting point. The information rate is equivalent to one planar cut every $\frac{4}{18}$ seconds.

\section{DISCUSSION}

We have taken four example frames from the film to illustrate the advantage of a film representation. The frames are taken at $l=53^{\circ}, l=120^{\circ}, l=180^{\circ}$, and 


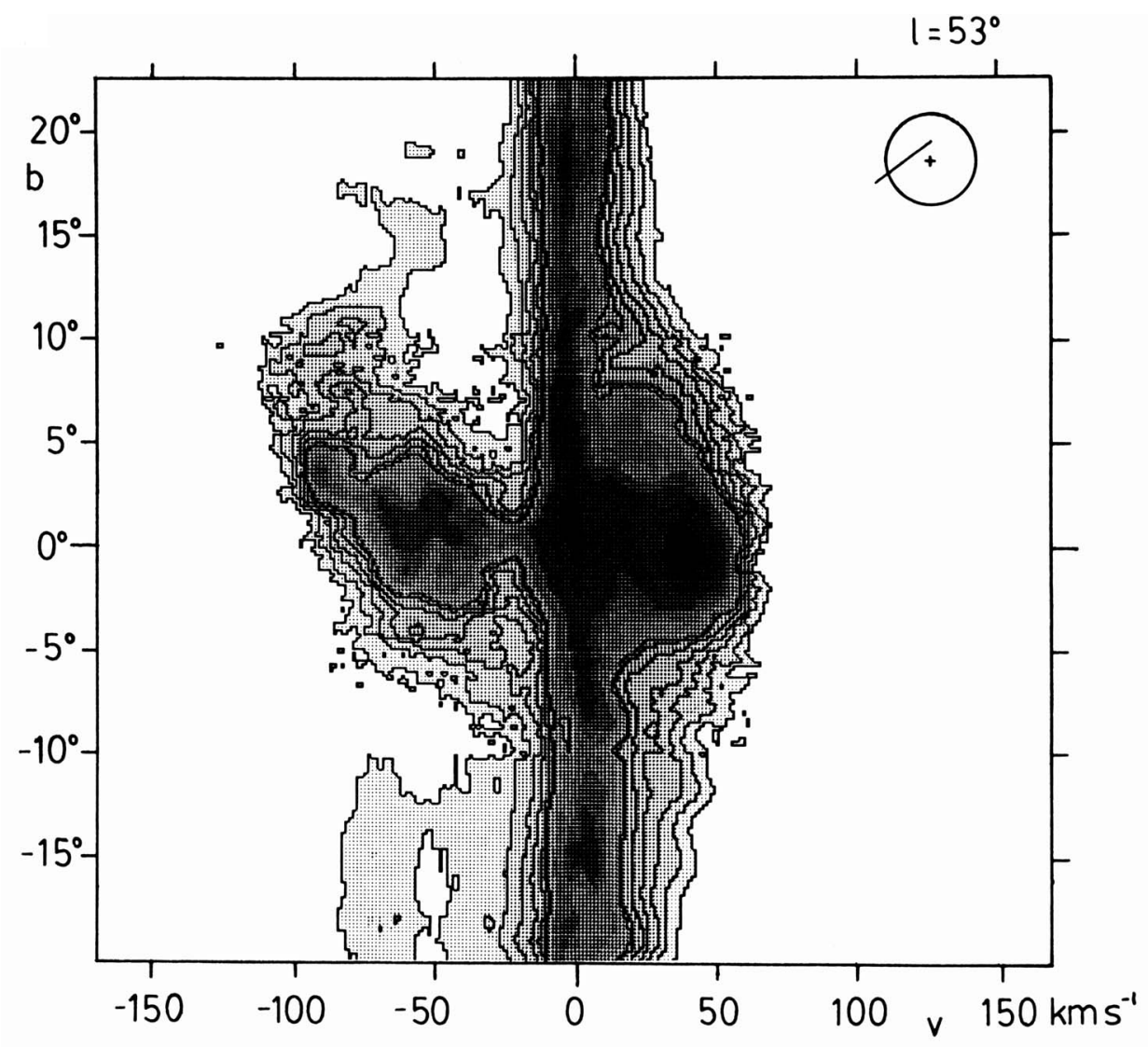

Figure 1. The sample frame from the film representation of the 3-dimensional HI dataset. The panel shows the HI column densities, scaled logarithmically, as a function of galactic latitude and velocity. The galactic longitude of the planar cut is shown by the line in the top view display of the galactic disk in the top right hand corner of the panel.

$l=220^{\circ}$ and are shown in figures 1 through 4 respectively. There are a number of interesting points that are immediately evident by looking at the film, these are $(i)$ the galactic rotation, $(i i)$ the warping of the galactic plane, (iii) the intermediate velocity clouds, $(i v)$ the filamentary structure of the neutral interstellar medium.

The galactic rotation manifests itself as the concentration of intensities near $v=0 \mathrm{~km} \mathrm{~s}^{-1}$ in figure 3 and the large excursions of emission at positive and negative velocities in figure 1,2 and 4. Assuming differential circular rotation and a homogeneous number density distribution the lines-of-sight at the low galactic latitude in figure 1 pass first through the inner part of the galaxy where the velocity vectors are pointing away from the observer. The line-of-sight continues through 


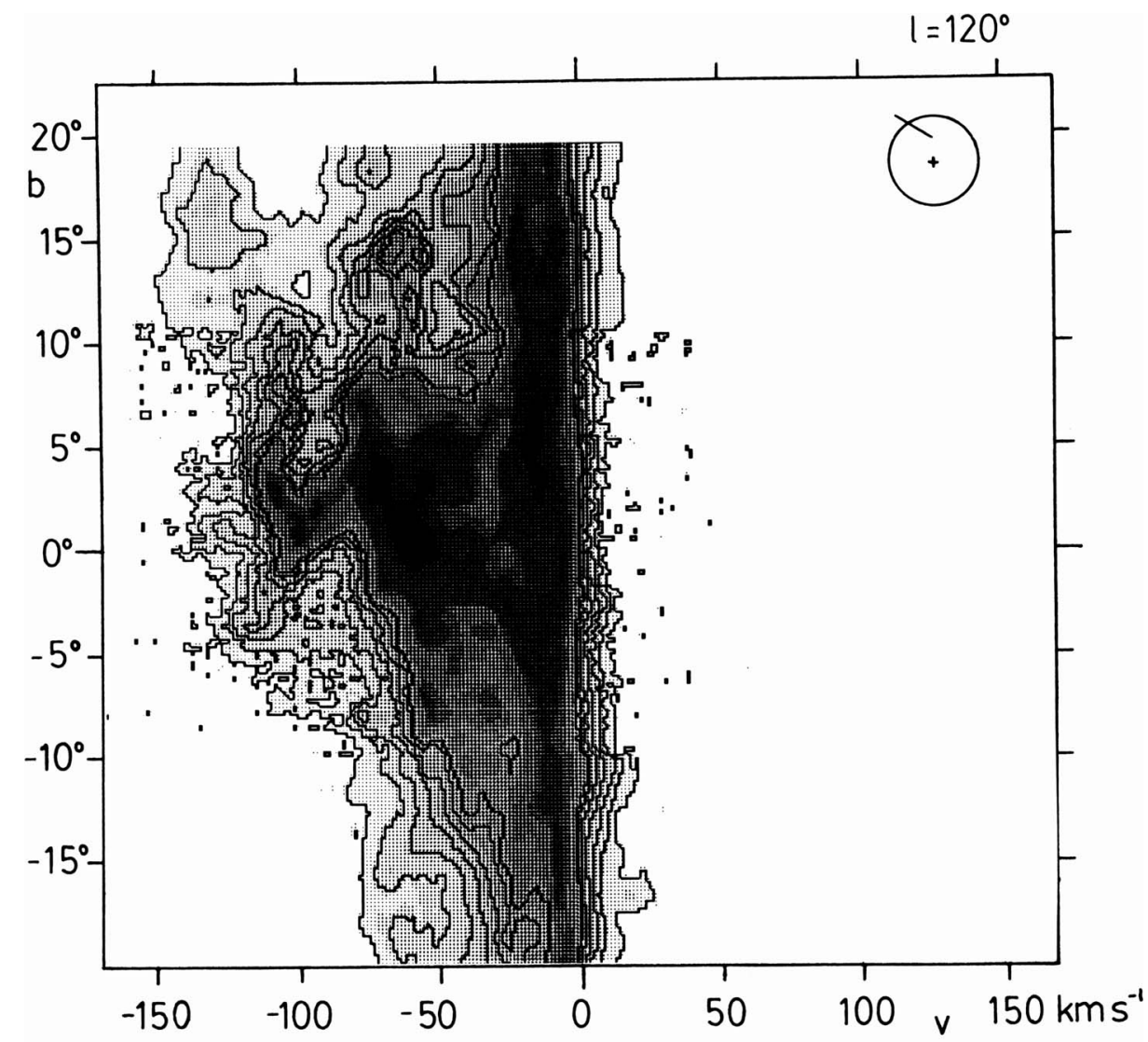

Figure 2. A frame from the film for the planar cut at $l=120^{\circ}$. The HI column densities are scaled logarithmically latitude and velocity. The galactic longitude of the planar cut is shown by the line in the top view display of the galactic disk in the top right hand corner of the panel. Note the signature of the warp as the positive offset of the mean of intensities at the negative velocities

the outer galaxy where the velocity vector point toward the observer. Therefore the figure 1 emission at positive radial velocities corresponds to material inside and the emission at negative radial velocities to material outside the solar circle. On a line-of-sight passing inside the solar circle there are two point for which the radial velocities are equal. This distance ambiguity causes the observed intensities to become twice as intense compared to those for the outer galaxy. For figure 2 the lines-of-sight at the low galactic latitudes pass through the outer galaxy only yielding negative radial velocities. The lines-of-sight at the low galactic latitudes corresponding to figure 4 also pass through the outer galaxy but now the velocity vectors are pointing away from the observer yielding positive radial velocities only. For figure 3 the velocity vectors are perpendicular to the line of sight causing all 


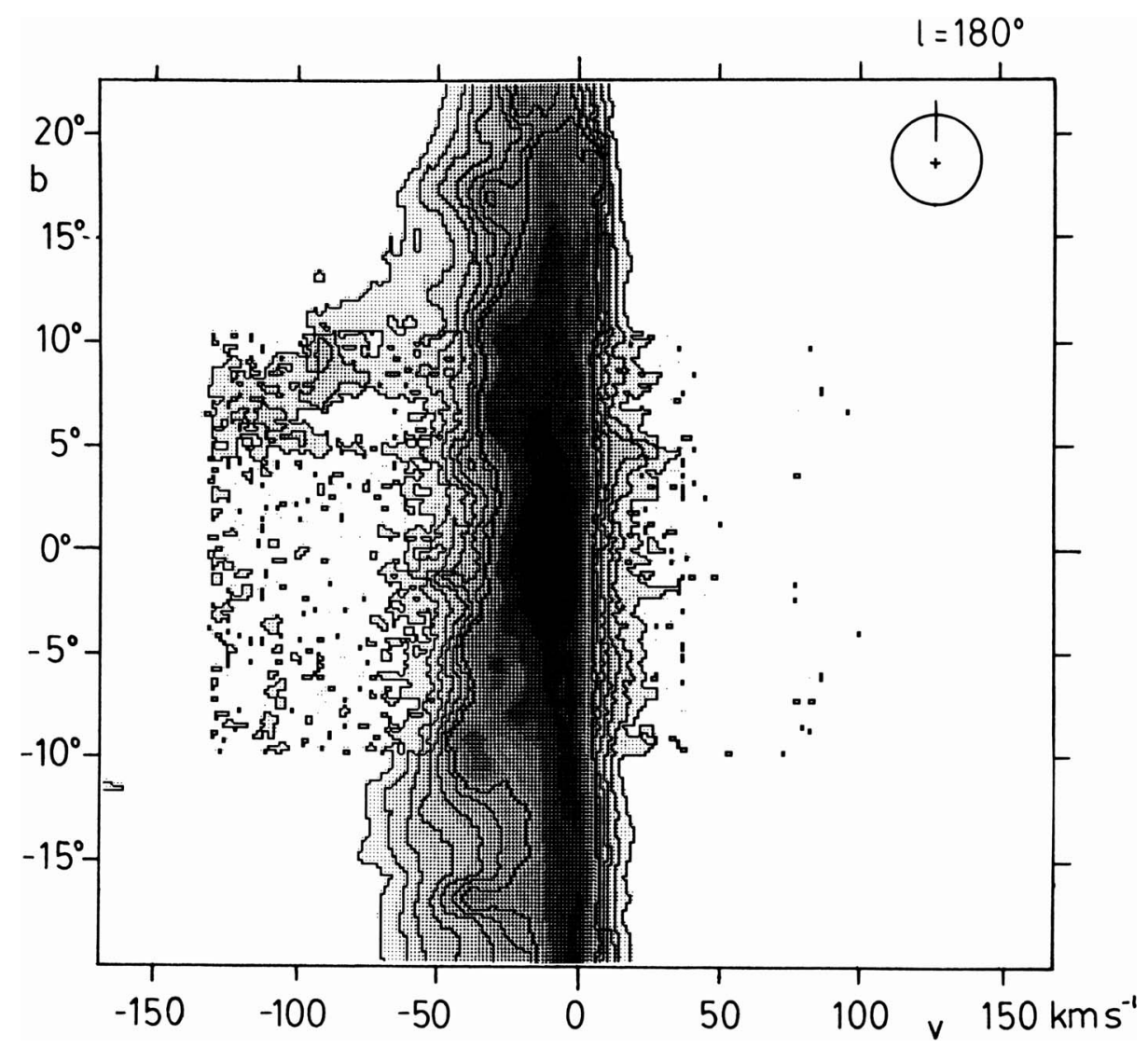

Figure 3. A frame from the film for the planar cut at $l=180^{\circ}$. Scaling of column densities is according to figure 1. Note the concentration of emission near $V_{\text {rad }}=0 \mathrm{~km} \mathrm{~s}^{-1}$. This is due to the fact that the line-of-sight is perpendicular to all the velocity vectors.

emission to occur near $V_{\text {rad }}=0 \mathrm{~km} \mathrm{~s}^{-1}$. The slight deviations from $0 \mathrm{~km} \mathrm{~s}^{-1}$ in this figure give a hint of the magnitude of non-circular motion of the neutral atomic hydrogen in that region. The emission in all figures near $0 \mathrm{~km} \mathrm{~s}^{-1}$ is caused by the HI material in the solar neighbourhood.

The warping of the galactic plane is evident in figures 1 and 2 by the positive displacement of the center of emission at the negative radial velocities, and to a lesser extend in figure 4 by the negative displacement at positive radial velocities. These regions in figures 1,2 and 4 correspond to material in the outer galaxy; the region in figure 1 corresponding to material in the inner galaxy shows no displacement. We may conclude that the warp of the galactic plane starts to become significant at $R>R_{\odot}$ and that the galactic plane inside $R=R_{\odot}$ is rather flat. A detailed description of the properties of the warp can be found in Burton (1987).

Clouds of neutral atomic hydrogen at higher galactic latitudes and interme- 


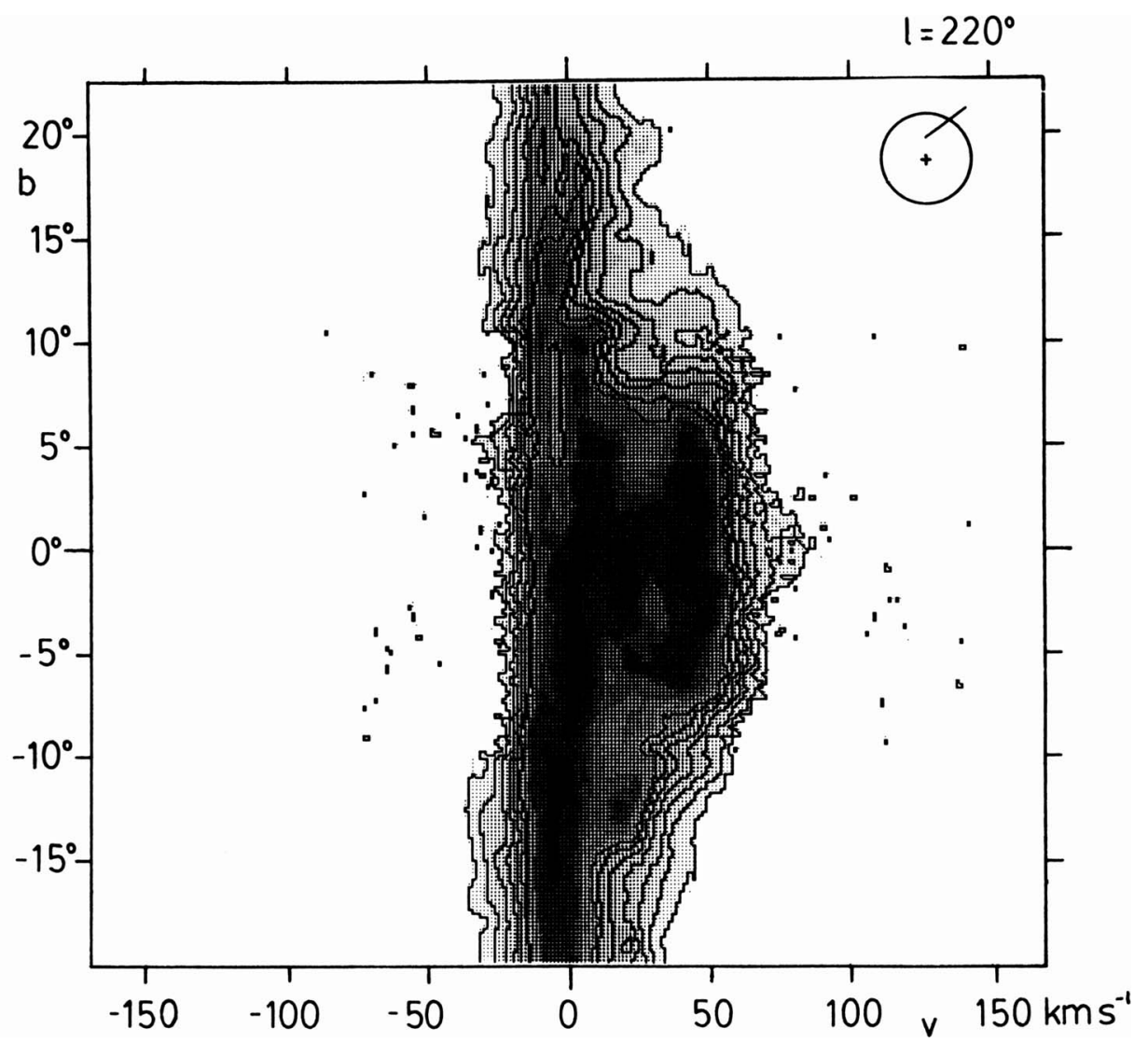

Figure 4. A frame from the film for the planar cut at $l=220^{\circ}$. Scaling of column densities is according to figre 1. The signature of galactic rotation is present by the emission at negative velocities in this figure, compared to the emission at positive velocities in figures 1 and 2 .

diate negative velocities are a member of the Intermediate Velocity Clouds (Hulsbosch, 1975). A few examples are present in the figures. In figure 2 the emission near $b=15^{\circ}, v=-130 \mathrm{~km} \mathrm{~s}^{-1} \mathrm{c}$ can be catalogued as an intermediate velocity cloud if the sensitivity of the HI survey at that point is not enough to show the connection of this cloud to the general warp signature. Numerous cases of previously identified, isolated intermediate velocity clouds are now clearly associated with material extending from the galactic plane. In figure 3 some less clearly defined structures near $b=8^{\circ}, v=-80 \mathrm{~km} \mathrm{~s}^{-1}$ are of identical nature. By increasing the sensitivity of the HI surveys it will become clear that large portions of isolated HI material are in fact embedded in low density $\mathrm{HI}$ environments that connect them to the galactic structure.

The filamentary structure of the neutral hydrogen distribution as depicted in the example frames from the film can be the result of two things. Either a fil- 


\begin{tabular}{|c|c|c|c|}
\hline Reference & Telescope & $\begin{array}{l}\text { Beam } \\
\text { (arc min) }\end{array}$ & $\begin{array}{l}\text { l-coverage } \\
0\end{array}$ \\
\hline Kepner (1970) & Dwingeloo 25-m & 36 & $48 \leq l \leq 228 ; \Delta l=4$ \\
\hline Weaver and Williams $(1973,1974 a)$ & Hat Creek 85-foot & 36 & $10 \leq l \leq 250 ; \Delta l=0.5$ \\
\hline Weaver and Williams (1974) & Hat Creek 85-foot & 36 & $12 \leq l \leq 249.5 ; \Delta l=2.5$ \\
\hline Heiles and Habing (1974) & Hat Creek 85-foot & 36 & $\begin{array}{l}\text { all } l \text { at }|b|>10 \\
\delta>-30 ; \Delta l=0.3 \cos b\end{array}$ \\
\hline $\begin{array}{l}\text { Colomb, Pöpel, and Heiles } \\
(1977,1980)\end{array}$ & IAR 100-foot & 30 & $\begin{array}{l}\text { all } l \text { at }|b|>10 \\
\delta<-25 ; \Delta \delta=1\end{array}$ \\
\hline Cleary, Heiles and Haslam (1979) & Parkes 60-foot & 48 & $\begin{array}{l}\text { all } l \text { at }|b|>10 \\
b>-25, \delta<-30 ; \Delta \delta=1\end{array}$ \\
\hline Kerr, Bower, and Henderson (1981) & Parkes 210-foot & 16 & $230 \leq l \leq 350 ; \Delta l=0.1$ \\
\hline Westerhout and Wendlandt (1982) & Green Bank 300-foot & 13 & $11 \leq l \leq 235 ; \Delta l=0.2$ \\
\hline $\begin{array}{l}\text { Strong, Riley, Osborne, and } \\
\text { Murray (1982) }\end{array}$ & Parkes 210-foot & 16 & $12>l>245 ; \Delta l=0.5$ \\
\hline Kerr, Bowers, Jackson, Kerr (1985) & Parkes 60-foot & 48 & $240 \leq l \leq 350 ; \Delta l=0.5$ \\
\hline Stark, Bally, Linke and Heiles & BTL 6-m horn & 150 & $\begin{array}{l}\text { all } l \text { at } \delta>-40 \\
\Delta l \sim 2\end{array}$ \\
\hline Burton (1986) & Green Bank 140-foot & 21 & $\begin{array}{l}\text { all } l \text { at } \delta>-46 \\
-20<b<20(33) ; \Delta l=1\end{array}$ \\
\hline
\end{tabular}

amentary density distribution or a small scale coherent velocity structure causes local intensity enhancements in the maps such that we see filaments in the data. Burton (1970) has shown that small systematic deviations from circular symmetry are already capable of creating the observed intensity variations. Deul and Burton (1987), however, have noted using the IRAS $100 \mu m$ observations that there is a strong correlation between the local HI column density and dust emissivities. Although infrared emissivities are influenced by the interstellar radiation field the observed $100 \mu m$ cirrus is intrinsically filamentary. This suggests that the HI material is also intrinsically filamentary of structure. 


\begin{tabular}{|c|c|c|c|}
\hline b-coverage & $\begin{array}{l}\mathrm{v} \text {-coverage } \\
\mathbf{k m ~ s}^{-1}\end{array}$ & $\begin{array}{l}3 \sigma \text { Sensitivity } \\
(\mathrm{K})\end{array}$ & Form of Display \\
\hline $6 \leq b \leq 20 ; \Delta b=2$ & $-220<v<0 ; \Delta v=10.6$ & 0.2 & Gaussian analysis \\
\hline$-10 \leq b \leq+10 ; \Delta b=2.5$ & $\begin{array}{l}\text { center vel. } \pm 100 ; \Delta v=2.1\left(\mathrm{~T}_{\mathrm{A}}>4 \mathrm{~K}\right) \\
\Delta v=6.3\left(\mathrm{~T}_{\mathrm{A}}<4 \mathrm{~K}\right)\end{array}$ & $\begin{array}{l}1.7\left(\mathrm{~T}_{\mathrm{A}}>4 \mathrm{~K}\right) \\
1.0\left(\mathrm{~T}_{\mathrm{A}}<4 \mathrm{~K}\right)\end{array}$ & $\begin{array}{l}\text { Spectra }(1973) \text { and } \\
\left.(b, v)\right|_{\iota} \text { maps }(1974 a)\end{array}$ \\
\hline $10<|b|<30 ; \Delta b=0.5$ & $\begin{array}{l}\text { center vel. } \pm 200 ; \Delta v=2.1\left(\mathrm{~T}_{\mathrm{A}}>4 \mathrm{~K}\right) \\
\Delta v=6.3\left(\mathrm{~T}_{\mathrm{A}}<4 \mathrm{~K}\right)\end{array}$ & $\begin{array}{l}1.7\left(\mathrm{~T}_{\mathrm{A}}>4 \mathrm{~K}\right) \\
1.0\left(\mathrm{~T}_{\mathrm{A}}<4 \mathrm{~K}\right)\end{array}$ & $\begin{array}{l}\text { spectra and } \\
\left.(b, v)\right|_{1} \text { maps }\end{array}$ \\
\hline all $|b|>10$ at $\delta>-30$ & $\begin{array}{l} \pm 50 \text { at } \Delta v=2.1 ; \text { to }-92 \text { and }+75 \text { at } \\
\Delta v=6.3\end{array}$ & $\sim 1.2$ & $\left.(l, v)\right|_{b}$ maps \\
\hline $\begin{array}{l}\text { all }|b|>10 \text { at } \delta<-25 \\
\Delta \delta=1\end{array}$ & $-38<v<38 ; \Delta v=2$ & 2 & $\left.(l, b)\right|_{0}$ maps \\
\hline $\begin{array}{l}|b|>10, b>-25 \text { at } \\
\delta<-30 ; \Delta \delta=1\end{array}$ & $-148<v<300 ; \Delta v=7.0$ & 0.3 & $\begin{array}{l}\text { representative } \\
\left.(l, b)\right|_{0} \text { maps }\end{array}$ \\
\hline$-2 \leq b \leq 2 ; \Delta b=0.1$ & center vel. $\pm 160 ; \Delta v=2.0$ & 2.0 & $\begin{array}{l}\left.(l, v)\right|_{b} \text { and } \\
\left.(b, v)\right|_{l} \text { maps }\end{array}$ \\
\hline$-2 \leq b \leq 2 ; \Delta b=0.1$ & center vel. $\pm 125 ; \Delta v=2.0$ & $\begin{array}{l}\sim 3(\text { lower } \delta) \\
\sim 1.5(\text { higher } \delta)\end{array}$ & $\left.(l, v)\right|_{b}$ maps \\
\hline$|b|<10 ; \Delta b=1$ & $-211<v<211 ; \Delta v=0.8$ & 3 & $(l, b)$ map total-v \\
\hline$|b|<10 ; \Delta b=0.25$ & center vel. $\pm 150 ; \Delta v=2$ & 0.8 & $\left.(b, v)\right|_{\iota}$ maps \\
\hline $\begin{array}{l}\text { all } b \text { at } \delta>-40 \\
\Delta b \sim 2\end{array}$ & center vel. $\pm 300 ; \Delta v=5.3$ & $<0.1$ & spectra \\
\hline $\begin{array}{l}-20 \leq b \leq 20(33) \text { at } \\
\delta>-46 ; \Delta b=1\end{array}$ & $\begin{array}{l}\text { center vel. } \pm 250 ; \Delta v=1.0\left(\mathrm{~T}_{\mathrm{A}}>0.5 \mathrm{~K}\right) \\
\Delta v=2.1\left(\mathrm{~T}_{\mathrm{A}}<0.5 \mathrm{~K}\right)\end{array}$ & $\begin{array}{l}0.2\left(\mathrm{~T}_{\mathrm{A}}>0.5 \mathrm{~K}\right) \\
0.14\left(\mathrm{~T}_{\mathrm{A}}<0.5 \mathrm{~K}\right)\end{array}$ & $\begin{array}{l}\left.\left.(l, v)\right|_{b} \&(b, v)\right|_{l} \text { map } \\
\left.(l, b)\right|_{v} \text { maps }\end{array}$ \\
\hline
\end{tabular}

\section{References}

Burton, W.B., 1970, Astron. Astrophys., 10, 76

Burton, W.B., 1986, Astron. Astrophys. Suppl., 63, 365

Burton, W.B., 1987, in "Galactic and Extragalactic Radio Astronomy", eds. G.L. Verschuur, K.I. Kellermann, Springer Verlag

Cleary, M.N., Heiles, C., Haslam, C.G.T., 1979, Astron. Astrophys. Suppl., 36, 95 Colomb, F.R., Pöppel, W.G.L., Heiles, C., 1977, Astron. Astrophys. Suppl., 29, 89 Colomb, F.R., Pöppel, W.G.L., Heiles, C., 1980, Astron. Astrophys. Suppl., 40, 47 
Deul, E.R., 1987, in "Comets to Cosmology", A. Lawrence ed., Springer, London Heiles, C., Habing, H.J., 1974, Astron. Astrophys. Suppl., 14, 1

Hulsbosch, A.N.M, 1975, Astron. Astrophys., 40, 1

Kalberla, P.M.W., Mebold, U., Reich, W., 1980, Astron. Astrophys., 82, 275

Kepner, M., 1970, Astron. Astrophys., 5,444

Kerr, F.J., Bowers, P.F., Henderson, A.P., 1981, Astron. Astrophys. Suppl., 44, 63

Kerr, F.J., Bowers, P.F., Jackson, P.D., Kerr, M., 1987, Astron. Astrophys. Suppl., 66, 373

Lockman, F.J., Jahoda, K., McCammon, D., 1986, Astrophys. J., 302, 432

Stark, A.A., Bally,J., Linke, R.A., Heiles, C., 1985, preprint

Strong, A.W., Riley, P.A., Osborne, J.L., Murray, J.D., 1982, Mon. Not. R. Astron. Soc., 201, 495

Weaver, H., Williams, D.R.W., 1973, Astron. Astrophys. Suppl., 8, 1

Weaver, H., Williams, D.R.W., 1974a, Astron. Astrophys. Suppl., 17, 1

Weaver, H., Williams, D.R.W., 1974b, Astron. Astrophys. Suppl., 17, 251

Westerhout, G., Wendlandt, H.-U., 1982, Astron. Astrophys. Suppl., 49, 137 
Discussion:

WIELEN

At the IAU General Asembly at New

Delhi in 1985, IAU Commission 33 proposed to use $R_{0}=8.5$ $\mathrm{kpc}$ as the new standard value for the distance of the sun from the galactic center. It may be useful for comparisons with other papers in the future, to present also figures using this new standard value of $R_{0}$.

DUEL

For comparison with published data I have used $R_{0}=10 \mathrm{kpc}$ here. Publication of our results

will, however, be based on $R_{0}=8.5 \mathrm{kpc}$. 